\title{
La cardiopatia ischemica nel paziente affetto da malattia renale cronica
}

\author{
Luca Di Lullo ${ }^{1}$, Fulvio Floccari ${ }^{2}$, Antonio De Pascalis ${ }^{3}$, Rodolfo Rivera ${ }^{4}$, Antonio Bellasi ${ }^{5}$, Alberto Santoboni ${ }^{1}$, \\ Francesca Santoboni ${ }^{1}$, Annalisa Villani ${ }^{6}$, Moreno Malagutit ${ }^{2}$, Claudio Ronco ${ }^{7}$
}

${ }^{1}$ U.O.C. Nefrologia e Dialisi, Ospedale "Parodi-Delfino", Colleferro (RM)

${ }^{2}$ U.O.C. Nefrologia e Dialisi, Ospedale "S. Paolo", Civitavecchia (RM)

${ }^{3}$ U.O.C. Nefrologia e Dialisi, Ospedale "V. Fazzi", Lecce

${ }^{4}$ Clinica Nefrologica, Ospedale "S. Gerardo", Monza

${ }^{5}$ U.O.C. Nefrologia e Dialisi, Ospedale "S. Anna", Como

${ }^{6}$ Reparto Nefrologia e Dialisi, Policlinico "L. Di Liegro", Roma

${ }^{7}$ International Renal Research Institute, Ospedale "S. Bortolo", Vicenza

\begin{abstract}
Coronary artery disease (CAD) in chronic kidney disease patients
There is a great deal of data demonstrating a close association between chronic kidney disease (CKD) and cardiovascular disease, particularly coronary artery disease (CAD). About one half of deaths in patients with end-stage renal disease (ESRD) are of cardiovascular causes. The association between CKD and ischemic heart disease can be explained in part because both conditions are related to the traditional risk factors for atherosclerotic vascular disease. However, CKD might also have a casual role in the development and progression of CAD, with several mechanisms potentially involved. Among CAD patients, those with CKD have systematically a worse prognosis than those without CKD irrespective of the clinical presentation or the treatments applied. Current therapy is based on small trials and observational data and on the extrapolation of the strategies that have been proved useful in the general population, although many treatments that have been shown to improve the prognosis of CAD patients are underused in those with renal impairment.
\end{abstract}

Keywords: Atherosclerosis, Chronic kidney disease, Coronary artery disease, Coronary revascularization

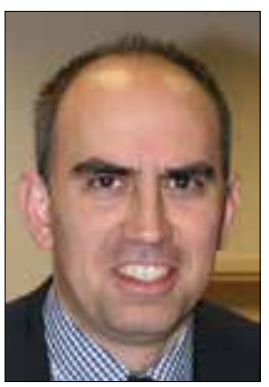

Luca Di Lullo

\section{Epidemiologia}

Allo stato attuale, la gran mole di dati disponibili in letteratura evidenzia una stretta correlazione tra malattia renale cronica (CKD) e patologia cardiovascolare, risultando, quest'ultima, la causa principale di mortalità e morbidità nei pazienti con danno renale.

Più in particolare, nell'ambito della patologia cardiovascolare, la parte del leone è giocata dalla malattia coronarica

Accepted: February 2, 2016

Published online: February 16, 2016

Indirizzo per la corrispondenza:

Dr. Luca Di Lullo

U.O.C. Nefrologia e Dialisi

Ospedale "L. Parodi-Delfino"

Piazza A. Moro, 1

00034 Colleferro (RM)

dilulloluca69@gmail.com con elevata incidenza sia di sindrome coronarica acuta sia di cardiopatia ischemica cronica $(1,2)$.

$\mathrm{Se}$, più in particolare, si osserva la popolazione di pazienti affetta da malattia renale cronica terminale (end-stage renal disease, ESRD), circa il 50\% dei decessi avviene per eventi cardiovascolari acuti $(1,2)$.

Nello studio di Go et al, che ha arruolato oltre un milione di pazienti con un periodo di follow-up di 2.84 anni, è stata studiata l'associazione tra stima del filtrato glomerulare (GFR) e rischio di morte, eventi cardiovascolari e giorni di ospedalizzazione (3).

II rischio relativo di morte per tutte le cause, l'incidenza di eventi cardiovascolari maggiori e la durata media del periodo di ospedalizzazione risultavano aumentati in maniera direttamente proporzionale alla riduzione del filtrato glomerulare (3).

Prendendo come riferimento i soggetti con eGFR $\geq 60 \mathrm{~mL} /$ $\mathrm{min} / 1.73 \mathrm{~m}^{2}$, il rischio di eventi cardiovascolari, definito come ricovero per sindrome coronarica acuta, scompenso cardiaco, ictus e/o malattia arteriosa periferica, varia da un incremento del $43 \%$ in soggetti con eGFR compreso tra 45 e $59 \mathrm{~mL} /$ $\mathrm{min} / 1.73 \mathrm{~m}^{2}$ a un incremento del $343 \%$ in coloro i quali presentano valori di eGFR $<15 \mathrm{~mL} / \mathrm{min} / 1.73 \mathrm{~m}^{2}$ (3).

L'associazione tra CKD e patologia cardiovascolare è stata 
TABELLA I - Valutazione del rischio cardiovascolare secondo le lineE guida ESC 2012

1. Rischio molto elevato. Soggetti che presentano una delle seguenti condizioni:

- CVD documentata da procedure invasive o non invasive, pregresso infarto del miocardio, pregresse procedure di rivascolarizzazione coronarica e/o arteriosa in generale, pregresso ictus, pregressa malattia arteriosa periferica

- diabete mellito (tipo 1 o tipo 2) in presenza di uno o più fattori di rischio cardiovascolare e/o segni di danno d'organo (p. es., microalbuminuria)

- $\quad$ CKD di grado severo (eGFR $<30 \mathrm{~mL} / \mathrm{min} / 1.73 \mathrm{~m}^{2}$ )

- uno SCORE calcolato $\geq 10 \%$ per rischio di CVD fatale a 10 anni

2. Rischio elevato. Soggetti che presentano una delle seguenti condizioni:

- $\quad$ presenza di singoli fattori di rischio cardiovascolare, purché di livello severo (dislipidemia severa, ipertensione arteriosa severa)

- diabete mellito (tipo 1 o tipo 2) in assenza di fattori di rischio cardiovascolare e segni di danno d'organo

- $\quad$ CKD di grado moderato (eGFR tra 30 e $59 \mathrm{~mL} / \mathrm{min} / 1.73 \mathrm{~m}^{2}$ )

- uno SCORE calcolato $\geq 10 \%$ per rischio di CVD fatale a 10 anni

3. Rischio moderato:

uno SCORE calcolato tra l' 1 e il 5\% per rischio di CVD fatale a 10 anni (potenzialmente modulato dalla presenza di fattori di rischio cardiovascolare come storia familiare di CAD precoce, obesità addominale, ridotta attività fisica, presenza di elevati livelli sierici di LDL-colesterolo, elevati livelli sierici di trigliceridi, elevati livelli di PCR ad alta sensibilità, fibrinogeno, omocisteina e apolipoproteina-B)

4. Basso rischio:

- uno SCORE calcolato <1\% per rischio di CVD fatale a 10 anni

CVD: malattia cardiovascolare; CKD: malattia renale cronica

osservata non solo nella popolazione generale ma anche nei pazienti ipertesi $(4,5)$ che presentano altri fattori di rischio come malattia cardiovascolare nota in fase di compenso (6) ovvero diabete mellito con macroalbuminuria (7).

Macro e microalbuminuria sono responsabili di un aumentato rischio cardiovascolare anche in presenza di livelli normali di escrezione di albumina $(8,9)$. Una metanalisi condotta su un numero considerevole di trial clinici ha confermato, inoltre, l'esistenza di un'associazione lineare tra escrezione urinaria di albumina e mortalità per cause cardiovascolari, indipendentemente dai livelli di filtrato glomerulare (4).

\section{Fisiopatologia}

L'associazione tra malattia renale cronica e malattia coronarica (CAD), allo stato attuale, continua a rimanere in parte oscura a causa del fatto che entrambe le patologie sono correlate ai fattori di rischio tradizionali della malattia vascolare su base aterosclerotica.

Allo stesso tempo, però, la malattia renale cronica sembrerebbe avere un ruolo importante nell'insorgenza e nella progressione della malattia coronarica, grazie a diversi meccanismi fisiopatologici potenzialmente coinvolti $(9,10)$.

In primo luogo, la presenza di microalbuminuria e la riduzione del valore del filtrato glomerulare sono stati associati a una condizione, flusso mediata, di inappropriata vasodilatazione, con aumento dei livelli sierici dei marcatori di danno endoteliale e/o di dimetilarginina asimmetrica (ADMA) e di altri inibitori dell'ossido nitrico-sintetasi, a testimonianza di come la presenza di CKD possa indurre disfunzione endoteliale (11-13).
In secondo luogo, la presenza di una condizione di infiammazione cronica, in parte conseguenza di un aumento dello stress ossidativo, è piuttosto comune nei pazienti affetti da CKD e potrebbe favorire l'ulteriore progressione dei processi aterosclerotici $(9,14)$.

Infine, la progressione della malattia renale cronica è correlata anche a un'iperattivazione del sistema reninaangiotensina (RAAS) e del sistema nervoso simpatico (SNS). Un'aumentata reattività di RAAS e SNS favorisce, a sua volta, la progressione della malattia coronarica sia contribuendo al peggioramento di una condizione ipertensiva sia agendo direttamente sui vasi coronarici $(9,15)$.

Una caratteristica peculiare della coronaropatia cronica nei pazienti affetti da CKD è rappresentata dalla spiccata propensione alla formazione di calcificazioni vascolari sia a livello dell'intima che della media dei vasi arteriosi.

Tali calcificazioni sono maggiormente documentabili negli stadi avanzati della malattia renale e l'eziologia va ricercata nella malattia renale stessa (iperparatiroidismo secondario) e nelle conseguenze legate alla terapia praticata $(9,16)$.

In considerazione dell'evidente associazione tra CKD e CAD e di una verosimile relazione biologica di tipo causa/effetto, la condizione di malattia renale cronica viene, allo stato attuale, considerata come fattore di rischio per eventi cardiovascolari acuti/cronici $(17,18)$ (Tab. I).

\section{Diagnosi}

Porre diagnosi di CAD nei pazienti affetti da CKD rappresenta una vera e propria sfida, sia in condizioni di acuzie che 
in condizioni di relativa stabilità emodinamica.

Una proporzione significativa di pazienti affetti da CKD con CAD di grado severo si presenta sovente asintomatica ovvero con sintomatologia piuttosto atipica e aspecifica (19).

Quanto appena descritto appare riconducibile, in primis, sia alla disfunzione del sistema nervoso simpatico sia alla frequente associazione tra CKD e diabete (20).

Un ulteriore elemento di difficoltà diagnostica risiede nel fatto che il tracciato elettrocardiografico è spesso inficiato da anomalie legate alla presenza di comorbidità cardiovascolari, quali la presenza, per esempio, di ipertrofia ventricolare sinistra, di alterazioni aspecifiche del tratto ST-T, di modificazioni dell'onda T e di ritardo di conduzione intraventricolare; le suddette alterazioni del tracciato ECG complicano, inoltre, l'attendibilità dei test da sforzo al cicloergometro (21).

Per quanto concerne il dosaggio di biomarker specifici di danno ischemico cardiaco, quello delle troponine ad alta sensibilità risulta essere il più utilizzato e il più importante per la valutazione di un dolore toracico di sospetta natura cardiaca (22).

Allo stesso tempo, però, il livello delle troponine è spesso superiore al normale nei pazienti affetti da CKD per i quali è bene considerare come normali valori ben superiori a quelli medi della popolazione generale ma, soprattutto, controllare l'andamento temporale dei valori stessi (23).

D'altro canto, i livelli di troponine sieriche sono in grado di predire la presenza di malattia coronarica e gli outcome cardiovascolari nei pazienti affetti da CKD anche in assenza di sospetto di sindrome coronarica acuta (24).

In aggiunta alla troponina, altri biomarker (copeptina, peptidi natriuretici) hanno evidenziato il loro valore diagnostico e/o prognostico nei pazienti con sospetta sindrome coronarica acuta ma, a oggi, non vi è un impiego routinario nella pratica clinica (22).

L'impiego del test da sforzo al cicloergometro per la diagnosi non invasiva di malattia ischemica cronica è poco utilizzato nei pazienti con malattia renale cronica a causa della ridotta tolleranza all'esercizio fisico (25).

Nei pazienti affetti da CKD, la Tomografia Computerizzata (TC) e l'ecocardiogramma da stress con dobutamina rappresentano le due tecniche di imaging "da stress" più comunemente impiegate, in quanto entrambe in grado sia di valutare l'ischemia indotta dallo stress (in questo caso di tipo farmacologico) sia di fornire un quadro attendibile della funzione ventricolare sinistra totale e regionale.

La sensibilità e la specificità delle suddette tecniche nell'individuare la presenza di una CAD in pazienti affetti da CKD sono piuttosto variabili, soprattutto nei soggetti in trattamento sostitutivo della funzione renale (25-27).

De Lima et al. ne hanno studiato l'accuratezza, nella diagnosi di ischemia e nella capacità di predire eventi avversi, in 126 pazienti ad alto rischio in lista d'attesa per trapianto di rene, riscontrando, allo stesso tempo, una sensibilità inferiore al $75 \%$ di entrambe le metodiche e un'associazione irrilevante con eventi cardiaci maggiori (28).

In antitesi a quanto appena descritto, una metanalisi di 12 studi clinici (per un totale di circa 900 pazienti coinvolti) condotti su pazienti affetti da ESRD ha documentato come la presenza di un'ischemia cardiaca indotta con dobutamina fosse un forte predittore di eventi cardiovascolari maggiori e mortalità (29).

Le medesime conclusioni sono arrivate da una seconda metanalisi condotta su 52 studi clinici su circa 7000 potenziali candidati al trapianto renale: in questo caso, veniva osservato come la TC cardiaca e l'ecostress con dobutamina fossero sovrapponibili alla coronarografia nel predire eventi cardiaci maggiori (30).

Le attuali Linee Guida AHA/ACC raccomandano che i test da stress non invasivi vadano presi in considerazione nei pazienti candidati al trapianto renale che non presentano una patologia cardiaca in fase acuta (26).

Altre tecniche di imaging cardiovascolare possiedono un potere diagnostico molto ridotto nei pazienti affetti da CKD (si veda l'angio-TC tradizionale) oppure vanno escluse per l'impiego di m.d.c. potenzialmente nefrotossici, come avviene nel caso della risonanza magnetica nucleare, per il rischio di andare incontro a un quadro di fibrosi miocardica da gadolinio.

\section{Prognosi}

Nell'ambito della popolazione affetta da CAD, il sottogruppo con CKD presenta una prognosi sicuramente peggiore rispetto ai pazienti con funzione renale conservata, indipendentemente dal quadro clinico e dalla tipologia di trattamento impiegato.

Report clinici evidenziano tassi di mortalità per infarto del miocardio più elevati nei pazienti affetti da CKD allo stadio V (31) e un rischio di complicanze via via maggiore con il progressivo declino della funzione renale (32-34).

Gli stessi risultati sono stati evidenziati per le sindromi coronariche cosiddette NSTEMI, ossia caratterizzate dall'assenza dell'elevazione del tratto ST-T; per rinforzare il concetto va detto come il livello sierico di creatinina faccia parte del Grace Score, il punteggio in grado di predire outcome clinici in pazienti ricoverati per sindrome coronarica acuta $(35,36)$.

Sebbene ancora non si sia giunti a una conclusione per spiegare le ragioni di una prognosi meno favorevole nei pazienti affetti da CKD, è altrettanto verosimile che un maggior numero di comorbidità, un quadro di coronaropatia più severo, un maggior rischio di emorragie e un approccio terapeutico poco aggressivo rappresentino tutti degli elementi in grado di contribuire a una maggiore fragilità del paziente nefropatico (37-39). 


\section{Terapia farmacologica}

Dal momento in cui la maggior parte dei trial randomizzati ha escluso i pazienti con CKD di grado severo, allo stato attuale ci sono evidenze piuttosto limitate per quanto concerne il trattamento farmacologico della CAD nei pazienti con danno renale.

Gli attuali protocolli terapeutici si basano su trial condotti su un numero ristretto di pazienti e su pochi dati provenienti da studi osservazionali (37).

\section{a) Farmaci ad azione anti-ischemica}

Approntare una terapia farmacologica ottimale risulta di fondamentale importanza nei pazienti affetti da CKD, per i quali non è sempre possibile ricorrere a procedure di rivascolarizzazione efficaci per il grado severo di CAD e per un rischio peri- e intra-procedurale notevolmente superiore a quello della popolazione generale.

I $\beta$-bloccanti riducono la richiesta di ossigeno a livello miocardico e, grazie a un effetto cronotropo negativo e al prolungamento della fase diastolica, determinano un miglioramento della perfusione coronarica risultando farmaci di prima linea nei pazienti affetti da CAD in fase di compenso (40).

Nell'ambito dei $\beta$-bloccanti, atenololo, nadololo, timololo e sotalolo sono molecole idrosolubili e vengono prevalentemente eliminate a livello renale, mentre metoprololo, propanololo, labetalolo, carvedilolo e nebivololo sono liposolubili e hanno un metabolismo prevalentemente epatico e sono da preferire in pazienti con una significativa riduzione della funzione renale.

I farmaci calcio-antagonisti rappresentano anch'essi dei farmaci di prima linea nel trattamento della CAD (40); più in particolare, i cosiddetti "diidropiridinici" (nifedipina, amlodipina e nicardipina) sono potenti vasodilatatori, mentre la sottoclasse dei "non-diidropiridinici" (verapamil e diltiazem) esercita la propria azione grazie agli effetti cronotropi e inotropi negativi in grado di ridurre il consumo di ossigeno a livello miocardico e di agire positivamente sui sintomi anginosi. I calcio-antagonisti possono essere utilizzati nei pazienti affetti da CKD grazie al metabolismo prevalentemente epatico (40).

I nitrati hanno guadagnato negli anni un posto di rilievo nel trattamento della CAD, in quanto sono in grado di indurre venodilatazione con conseguente vasodilatazione a livello del circolo coronarico.

La nitroglicerina, somministrata per via sublinguale, produce un sollievo immediato dai sintomi anginosi e presenta un buon profilo di sicurezza nei pazienti con CKD, sebbene possa indurre ipotensione nell'immediato periodo postdialitico, effetto favorito anche dalla deplezione di volume successiva alla seduta emodialitica (41). I nitrati a lunga durata d'azione presentano anch'essi un buon profilo di tollerabilità nei pazienti con malattia renale e non sono necessari aggiustamenti del dosaggio (41).
L'ivabradina è un farmaco di relativa nuova immissione sul mercato ed è in grado di inibire le correnti If presenti a livello del nodo seno-atriale, determinando una riduzione della frequenza cardiaca e un miglioramento della sintomatologia anginosa. Può essere impiegata in luogo oppure in associazione con i $\beta$-bloccanti, ma il suo utilizzo dovrebbe essere evitato nei pazienti in terapia con verapamil e/o diltiazem (42). Allo stato attuale sono a disposizione pochi dati clinici relativi all'efficacia e alla sicurezza d'impiego nei pazienti affetti da CKD; per tale motivo, la prescrizione di ivabradina va effettuata con cautela in questa popolazione di pazienti (42).

La ranolazina, al contrario dell'ivabradina, provoca un miglioramento della sintomatologia anginosa inibendo la fase tardiva del potenziale d'azione legato alle correnti del sodio e non determina alcun effetto emodinamico (43).

La ranolazina è metabolizzata a livello epatico ed eliminata per via renale; per tale motivo è consigliabile prestare attenzione al dosaggio nei pazienti con CKD allo stadio II-III ed evitarne l'impiego in coloro i quali presentano un valore di filtrato glomerulare $<30 \mathrm{~mL} / \mathrm{min} / 1.73 \mathrm{~m}^{2}$ (43).

II nicorandil è un farmaco vasodilatatore che agisce come un nitrato grazie all'effetto sui canali del potassio; viene metabolizzato ed escreto a livello renale ma può essere utilizzato senza modificazioni della posologia anche nei pazienti con severa compromissione della funzione renale (44).

La trimetazidina presenta ancora diversi lati oscuri e può essere considerata un farmaco antianginoso di seconda-terza scelta; è controindicata nei pazienti con CKD allo stadio $\mathrm{V}$ e andrebbe utilizzata con cautela nei pazienti con CKD allo stadio III-IV (45).

La trimetazidina potrebbe esercitare un effetto protettivo nei confronti della nefropatia da mezzo di contrasto nei pazienti con CKD allo stadio II-III (45).

\section{b) Farmaci antiaggreganti}

I farmaci antiaggreganti rappresentano uno dei capisaldi del trattamento farmacologico della CAD, sia nello stadio cronico della malattia (in monoterapia) sia in fase acuta, successivamente a un episodio infartuale ovvero nel periodo post-intervento di angioplastica percutanea (solitamente in associazione con altri farmaci antiaggreganti).

La presenza di un deficit della funzionalità renale diventa una discriminante importante, in quanto si associa a un rischio maggiore di eventi trombotici e di emorragia (46).

Una recente metanalisi effettuata in sottopopolazioni di pazienti affetti da CKD su 36 trials clinici riguardanti sia pazienti con sindrome coronarica acuta (SCA) sottoposti ad angioplastica percutanea $(\mathrm{PCl})$, sia pazienti con anamnesi negativa per cardiopatia, ha evidenziato come l'aumento del rischio emorragico e/o una terapia antiaggregante particolarmente aggressiva potrebbero determinare effetti che vanno ben al di là dell'azione antitrombotica nei pazienti affetti da CKD (47). 
Eventuali aggiustamenti posologici nei pazienti affetti da malattia renale cronica vanno effettuati seguendo quanto riportato nei documenti delle linee guida ESC del 2012 (22).

Tra i farmaci antiaggreganti, l'acido acetilsalicilico è di gran lunga il più impiegato; dati provenienti da studi osservazionali e trial clinici suggeriscono che risulta in grado di ridurre le complicanze ischemiche senza comportare una più elevata incidenza di fenomeni emorragici nei pazienti affetti da CKD, indipendentemente dal grado di severità della malattia renale (48-50).

Il clopidogrel, farmaco antiaggregante antagonista recettoriale della glicoproteina di membrana $\mathrm{P} 2 \mathrm{Y} 12$, di più recente introduzione sul mercato, riduce, soprattutto in associazione al salicilato, il rischio di eventi ischemici nel primo anno successivo a un evento coronarico acuto.

In una sottoanalisi nel trial CURE (Clopidrogrel in Unstable Angina to Prevent Recurrent Events), il clopidogrel si è rivelato in grado di ridurre il rischio di eventi ischemici nei pazienti con diversi gradi di malattia renale cronica con un incremento della percentuale di fenomeni emorragici assolutamente non correlati al grado di deficit della funzione renale (51).

Il prasugrel, rispetto al clopidogrel, è un più potente antagonista recettoriale del P2Y12 e i livelli plasmatici del suo metabolita attivo diminuiscono significativamente nei pazienti con malattia renale cronica terminale, pur non impattando in maniera negativa sull'attività antiaggregante (46).

In un recente trial randomizzato (che ha il suo limite nell'arruolamento di un ridotto numero di pazienti affetti da CKD), il prasugrel ha ridotto gli eventi ischemici rispetto a quanto ottenuto con il clopidogrel in pazienti con sindrome coronarica acuta trattati con $\mathrm{PCl}$, pur pagando qualcosa in termini di aumentato rischio emorragico (52).

In un altro studio che ha messo a confronto l'effetto di clopidogrel e prasugrel in pazienti trattati con sola terapia farmacologica post-infarto del miocardio, non sono stati documentati effetti collaterali di tipo emorragico nei pazienti con ridotta funzionalità renale (53).

Il ticagrelor è un altro potente inibitore del P2Y12 che, rispetto al clopidogrel, si è rivelato in grado di ridurre gli eventi ischemici e la mortalità, incrementando, al contempo, il rischio di fenomeni emorragici in pazienti affetti da SCA e non sottoposti a intervento di by-pass aorto-coronarico, come documentato nel trial PLATO, che non includeva pazienti in trattamento dialitico (54).

Nel suddetto trial, il $21 \%$ dei pazienti presentava vari gradi di coinvolgimento renale e la riduzione degli eventi ischemici dovuta al ticagrelor era percentualmente maggiore che nei pazienti con funzione renale conservata (55).

Infine, gli inibitori della glicoproteina Ilb/Illa associati alla doppia terapia antiaggregante sembrano poter aggiungere ulteriore protezione nei confronti degli eventi trombotici, a costo dell'aumentata incidenza del rischio emorragico nella popolazione generale e nei pazienti affetti da CKD, per i quali i dosaggi vanno sensibilmente ridotti (fino al 50\%) (22).

\section{c) Farmaci anticoagulanti e trombolitici}

Al pari degli antiaggreganti, i farmaci anticoagulanti risultano essere di fondamentale importanza per il trattamento della sindrome coronarica acuta, dal momento che essi riducono in maniera significativa i rischi di eventi ischemici.

L'eparina, nella sua forma non frazionata (UFH), si lega alle cellule endoteliali e viene metabolizzata dai macrofagi, configurandosi, in linea teorica, come l'anticoagulante ideale per i pazienti affetti da CKD, in quanto non è necessaria alcuna modificazione del dosaggio.

L'enoxaparina è caratterizzata da una prevalente clearance renale e, pertanto, sono necessari degli aggiustamenti posologici, in presenza di una severa compromissione della funzionalità renale (22).

L'enoxaparina si è rivelata in grado di ridurre l'incidenza di eventi ischemici nei pazienti con sindrome coronarica acuta con o senza sopraslivellamento del tratto ST-T. Per quanto riguarda i pazienti affetti da CKD, le informazioni sono piuttosto limitate, in quanto sono spesso esclusi dai grandi trial clinici, soprattutto in presenza di una grave compromissione della funzione renale.

Alcuni studi clinici non hanno riscontrato interazioni significative, per quanto riguarda eventi ischemici e fenomeni emorragici, tra trattamento farmacologico impiegato e funzione renale (56); altri dati, provenienti da ulteriori trial, hanno evidenziato come gli effetti benefici dell'enoxaparina, rispetto all'eparina non frazionata, fossero limitati ai pazienti con funzione renale conservata (57).

II fondaparinux è un'eparina di sintesi in grado di inibire selettivamente il fattore $\mathrm{Xa}$, che viene eliminato prevalentemente per via renale e che è controindicato nei pazienti con valore di eGFR $<20 \mathrm{~mL} / \mathrm{min} / 1.73 \mathrm{~m}^{2}$.

II trial OASIS-5, che ha escluso i pazienti con valore di creatinina sierica $\geq 3 \mathrm{mg} / \mathrm{dL}$, ha evidenziato la non inferiorità del fondaparinux nei confronti dell'enoxaparina nei pazienti con infarto del miocardio NSTEMI e valori di eGFR $<58 \mathrm{~mL}$ / $\mathrm{min} / 1.73 \mathrm{~m}^{2}$, a testimonianza del fatto che potrebbe trattarsi di un farmaco con un appeal significativo nei pazienti affetti da CKD di grado moderato (58).

La bivalirudina è un inibitore sintetico della trombina caratterizzato da una significativa clearance renale e, pertanto, è controindicata nei pazienti con ESRD (46). In diversi studi clinici ha presentato un'azione anti-trombotica simile a quella esercitata dalla combinazione eparina/inibitori della glicoproteina IIIb/IIla nei pazienti con sindrome coronarica acuta con e senza alterazione del tratto ST-T trattati con PCl, anche in presenza di un quadro di CKD di grado moderato (59).

\section{d) Altri farmaci impiegati in prevenzione secondaria}

Diversi studi clinici hanno documentato come i pazienti con CAD e CKD e, in particolar modo, quelli con ESRD non siano adeguatamente trattati dal punto di vista della prevenzio- 
ne secondaria, assumendo dosi inadeguate di farmaci come aspirina, statine, ACE-inibitori e $\beta$-bloccanti (60-62).

Diversi trial hanno valutato l'efficacia degli ACE-inibitori (ACEi) e degli antagonisti recettoriali dell'angiotensina II (AT-II antagonisti) nella prevenzione secondaria degli eventi cardiovascolari ma, sfortunatamente, non hanno mai preso in considerazione i pazienti affetti da CKD.

Studi clinici, anche se limitati dal punto di vista del campione numerico, effettuati con fosinopril ne hanno dimostrato l'efficacia in tema di prevenzione primaria (6).

Il trial HOPE, che ha escluso pazienti con valori di creatinina sierica $>2.3 \mathrm{mg} / \mathrm{dL}$, ha evidenziato il ruolo del ramipril nel ridurre l'endpoint composito per morte cardiovascolare, ictus e infarto del miocardio in una popolazione di pazienti affetti da arteriopatia periferica e CKD (6).

Sono disponibili poche informazioni per quanto riguarda l'eventuale ruolo protettivo degli AT-II antagonisti nei pazienti affetti da CAD e CKD (62-64).

Sebbene diversi studi clinici abbiano documentato outcome cardiovascolari più favorevoli nei pazienti in cui venivano ridotti in maniera piuttosto aggressiva i livelli di LDL-colesterolo, due trial randomizzati condotti su pazienti in trattamento emodialitico non hanno evidenziato un impatto favorevole di atorvastatina e rosuvastatina sull'incidenza di eventi ischemici $(65,66)$.

Lo studio SHARP (trial randomizzato che ha arruolato oltre 9000 pazienti affetti da CKD, dei quali oltre 3000 sottoposti a trattamento dialitico), condotto su pazienti con anamnesi negativa per infarto del miocardio e precedenti interventi di rivascolarizzazione coronarica, ha permesso di sottolineare I'impatto favorevole dell'associazione terapeutica ezetimibe/ simvastatina sia sui pazienti affetti da CKD in terapia conservativa sia sui pazienti sottoposti a trattamento dialitico (67-69).

\section{Rivascolarizzazione coronarica}

Al momento sono diverse le domande rimaste senza risposta in merito agli effetti benefici evidenziati dalla rivascolarizzazione coronarica nei pazienti affetti da CKD, sia per quanto riguarda la tempistica dell'intervento sia per l'approccio dal punto di vista tecnico.

Sebbene sia l'intervento di by-pass aorto-coronarico sia l'approccio percutaneo siano associati a un aumento significativo dell'incidenza di complicanze a breve termine $(70,71)$, studi di popolazione suggeriscono come i pazienti affetti da CKD possano trarre beneficio dalla rivascolarizzazione coronarica (72-75).

Nella Tabella II sono evidenziate le attuali raccomandazioni della Società Europea di Cardiologia in tema di rivascolarizzazione nei pazienti affetti da CKD (76).

\section{a) Rivascolarizzazione nei diversi contesti clinici di CKD}

La presenza di CKD di grado lieve-moderato (stadio I-IIIb)
TABELLA II - Raccomandazioni per la terapia di rivascolarizzazione in pazienti con CKD moderata e severa in accordo con le linee guida ESC in materia di rivascolarizzazione miocardica (71)

- L'intervento di CABG dovrebbe essere preso in considerazione (invece della $\mathrm{PCI}$ ) nei pazienti con CAD polidistrettuale e presenza di sintomi clinici/ischemia miocardica, nei quali il rischio chirurgico risulta essere accettabile e l'aspettativa di vita è superiore a 1 anno (raccomandazione di classe lla, livello di evidenza B)

- La $\mathrm{PCl}$ dovrebbe essere presa in considerazione invece dell'intervento di CABG nei pazienti con CAD polidistrettuale e presenza di sintomi clinici/ischemia miocardica, nei quali il profilo di rischio chirurgico è elevato ovvero l'aspettativa di vita è inferiore a 1 anno (raccomandazione di classe lla, livello di evidenza B)

- L'intervento di CABG, dopo l'esecuzione di un esame coronarografico, dovrebbe essere ritardato fino all'attenuazione dell'effetto del mezzo di contrasto iodato sulla funzione renale (raccomandazione di classe lla, livello di evidenza B)

- Nel caso venga posta l'indicazione al posizionamento di stent intra-coronarici, andrebbero preferiti quelli medicati (raccomandazione di classe I, livello di evidenza B)

CABG: by-pass aorto-coronarico, CAD: malattia arteriosa coronarica; CKD: malattia renale cronica; $\mathrm{PCl}$ : angioplastica coronarica percutanea

non risulta essere associata a un'aumentata incidenza di restenosi dopo l'angioplastica in pazienti con CAD in fase di compenso emodinamico (77).

Un'analisi post-hoc di un gruppo di pazienti affetti da CKD (stadio II-IIla) con CAD stabilizzata nell'ambito dello studio COURAGE ha evidenziato come, in maniera similare alla popolazione con funzione renale conservata, l'associazione di $\mathrm{PCl}$ e terapia farmacologica non offra ulteriori vantaggi, in termini di sopravvivenza e di protezione dal re-infarto, rispetto alla sola terapia farmacologica (78). Alla luce di queste evidenze e considerando il rischio della nefropatia da mezzo di contrasto, l'esecuzione di una coronarografia dovrebbe essere attentamente valutata nei pazienti affetti da CAD con malattia renale (78).

Pareri tuttora discordanti vi sono circa l'indicazione di un trattamento invasivo nei pazienti con infarto del miocardio NSTEMI e CKD, probabilmente a causa del ridotto numero di studi randomizzati.

Un registro nazionale comprendente oltre 23000 pazienti con infarto del miocardio NSTEMI, dei quali il $25 \%$ circa affetto da CKD di diversa gravità, ha sottolineato come la rivascolarizzazione precoce fosse associata a un più basso tasso di mortalità a 1 anno nei pazienti con CKD allo stadio II-IIIb che nei soggetti in trattamento dialitico (75).

Una metanalisi più recente, condotta su oltre 20000 pazienti con diagnosi di infarto miocardico NSTEMI e CKD, ha evidenziato come vi fosse una correlazione positiva tra procedure di rivascolarizzazione precoce e sopravvivenza a un anno, indipendentemente dallo stadio di CKD (73).

Non vi sono, al momento, dati riguardanti gli outcome 
cardiovascolari nei pazienti con CKD e infarto miocardico con sopraslivellamento del tratto ST-T (STEMI).

\section{b) Angioplastica percutanea (PCI) vs rivascolarizzazione chirurgica (CABG)}

Nei pazienti con interessamento di una singola arteria coronaria (esclusa la dominante sinistra), il trattamento percutaneo risulta essere preferibile a quello di by-pass aortocoronarico. Nei pazienti con malattia coronarica bi-trivasale, la decisione terapeutica dovrebbe competere a un team multidisciplinare, compreso lo specialista nefrologo, nel caso in cui si tratti di un paziente con CKD (76).

Sebbene non vi siano trial randomizzati in grado di fornire risposte definitive sulla migliore strategia di rivascolarizzazione nei pazienti con CKD, alcuni dati provenienti da studi osservazionali possono fornire interessanti risposte.

In dati provenienti da registri nazionali riguardanti pazienti affetti da CAD sottoposti a coronarografia, l'esecuzione di CABG era associata a percentuali di sopravvivenza migliori sia in pazienti con funzione renale conservata sia in pazienti affetti da CKD, laddove la PCl offriva risultati migliori, se confrontata con la terapia farmacologica, nei pazienti con ESRD e sottoposti a trattamento dialitico (72).

Un'analisi secondaria del trial ARTS (Arterial Revascularization Therapies Study), nel quale i pazienti con CAD trivasale venivano randomizzati e assegnati al trattamento $\mathrm{PCl}$ ovvero $C A B G$, non ha evidenziato differenze in termini di mortalità, infarto del miocardio e ictus a 3 anni ma percentuali più elevate di ricorso a una nuova $\mathrm{PCl}(79,80)$.

Nella popolazione di pazienti con ESRD, l'analisi dei registri nazionali di pazienti affetti da CAD con follow-up medio di 5 anni non ha dimostrato significative differenze tra CABG e PCI per quanto riguarda la mortalità per tutte le cause, mentre era più frequente l'incidenza di morte cardiaca improvvisa e di sindrome coronarica acuta nel gruppo trattato con $\mathrm{PCl}(81)$.

Un'altra metanalisi di studi retrospettivi ha concluso che, anche se associata con un incremento del tasso di mortalità a breve termine, la CABG si dimostrava superiore alla $\mathrm{PCl}$ in termini di sopravvivenza a lungo termine con una minore incidenza, nel tempo, di episodi infartuali e la necessità di ulteriori procedure di rivascolarizzazione (82).

Le Linee Guida della Società Europea di Cardiologia riguardanti le procedure di rivascolarizzazione raccomandano l'esecuzione di CABG, rispetto alla $\mathrm{PCl}$, nei pazienti affetti da CKD con CAD multivasale sintomatica allorché il rischio operatorio sia sostenibile e l'aspettativa di vita del paziente sia superiore a un anno (76).

\section{Disclosures}

Financial support: No financial support was received for this submission.

Conflict of interest: The authors have no conflict of interest.

\section{Bibliografia}

1. Tonelli M, Wiebe N, Culleton B, et al. Chronic kidney disease and mortality risk: a systematic review. J Am Soc Nephrol 2006;17:2034-47.

2. United States Renal Data System. 2014 Annual Data Report. http://www.usrds.org/adr.aspx.

3. Go AS, Chertow GM, Fan D, McCulloch CE, Hsu CY. Chronic kidney disease and the risks of death, cardiovascular events, and hospitalization. N Engl J Med 2004;35:1296-305.

4. Matsushita K, van der Velde M, Astor BC, et al. Association of estimated glomerular filtration rate and albuminuria with all-cause and cardiovascular mortality in general population cohorts: a collaborative meta-analysis. Lancet 2010;375:2073-81.

5. Ruilope LM, Salvetti A, Jamerson K, et al. Renal function and in tensive lowering of blood pressure in hypertensive participants of the hypertension optimal treatment (HOT) study. J Am Soc Nephrol 2001;12:218-25.

6. Mann JF, Gerstein HC, Pogue J, Bosch J, Yusuf S. Renal insufficiency as a predictor of cardiovascular outcomes and the impact of ramipril: the HOPE randomized trial. Ann Intern Med 2001;134:629-36.

7. de Zeeuw D, Remuzzi G, Parving HH, et al. Albuminuria, a therapeutic target for cardiovascular protection in type 2 diabetic patients with nephropathy. Circulation 2004;110:921-7.

8. Mann JF, Yi QL, Gerstein HC. Albuminuria as a predictor of cardiovascular and renal outcomes in people with known atherosclerotic cardiovascular disease. Kidney Int Suppl 2004;92:S59-62.

9. Schiffrin EL, Lipman ML, Mann JF. Chronic kidney disease: effects on the cardiovascular system. Circulation 2007;116:85-97.

10. Hajhosseiny R, Khavandi K, Goldsmith DJ. Cardiovascular disease in chronic kidney disease: untying the Gordian knot. Int J Clin Pract 2013;67:14-31.

11. Vallance $\mathrm{P}$, Leone A, Calver A, Collier J, Moncada S. Accumulation of an endogenous inhibitor of nitric oxide synthesis in chronic renal failure. Lancet 1992;339:572-5.

12. Stehouwer CD, Henry RM, Dekker JM, Nijpels G, Heine RJ, Bouter LM. Microalbuminuria is associated with impaired brachial artery, flow-mediated vasodilation in elderly individuals without and with diabetes: further evidence for a link between microalbuminuria and endothelial dysfunction--the Hoorn Study. Kidney Int Suppl 2004;92:S42-4.

13. Stam F, van Guldener C, Becker A, et al. Endothelial dysfunction contributes to renal function-associated cardiovascular mortality in a population with mild renal insufficiency: the Hoorn study. J Am Soc Nephrol 2006;17:537-45.

14. Jofré R, Rodriguez-Benitez $P$, López-Gómez JM, Pérez-Garcia R. Inflammatory syndrome in patients on hemodialysis. J Am Soc Nephrol 2006;17 (Suppl. 3):S274-80.

15. Converse RL Jr, Jacobsen TN, Toto RD, et al. Sympathetic overactivity in patients with chronic renal failure. $\mathrm{N}$ Engl J Med 1992;327:1912-8.

16. Ketteler M, Schlieper G, Floege J. Calcification and cardiovascular health: new insights into an old phenomenon. Hypertension 2006;47:1027-34.

17. Catapano AL, Reiner Z, De Backer G, et al. ESC/EAS Guidelines for the management of dyslipidaemias The Task Force for the management of dyslipidaemias of the European Society of Cardiology (ESC) and the European Atherosclerosis Society (EAS). Atherosclerosis 2011;217:3-46.

18. Perk J, De Backer G, Gohlke H, et al. European Guidelines on cardiovascular disease prevention in clinical practice (version 2012). The Fifth Joint Task Force of the European Society of Cardiology and Other Societies on Cardiovascular Disease Prevention in Clinical Practice (constituted by representatives of nine 
societies and by invited experts). Eur Heart J 2012;33:1635-701 [Erratum in: Eur Heart J 2012;33:2126].

19. Braun WE, Phillips DF, Vidt DG, et al. Coronary artery disease in 100 diabetics with end-stage renal failure. Transplant Proc 1984;16:603-7.

20. Canto JG, Fincher $\mathrm{C}$, Kiefe $\mathrm{Cl}$, et al. Atypical presentations among Medicare beneficiaries with unstable angina pectoris. Am J Cardiol 2002;90:248-53.

21. Abe S, Yoshizawa M, Nakanishi N, et al. Electrocardiographic abnormalities in patients receiving hemodialysis. Am Heart J 1996;131:1137-44.

22. Hamm CW, Bassand JP, Agewall S, et al. ESC Guidelines for the management of acute coronary syndromes in patients presenting without persistent ST-segment elevation: The Task Force for the management of acute coronary syndromes (ACS) in patients presenting without persistent ST-segment elevation of the European Society of Cardiology (ESC). Eur Heart J 2011;32:2999-3054.

23. Di Lullo L, Barbera V, Santoboni A, et al. Troponins and chronic kidney disease. G Ital Nefrol 2015;32(4).

24. De Filippi C, Wasserman S, Rosanio S, et al. Cardiac troponin $\mathrm{T}$ and C-reactive protein for predicting prognosis, coronary atherosclerosis, and cardiomyopathy in patients undergoing long-term hemodialysis. JAMA 2003;290:353-9.

25. Karthikeyan V, Ananthasubramaniam K. Coronary risk assessment and management options in chronic kidney disease patients prior to kidney transplantation. Curr Cardiol Rev 2009;5:177-86.

26. Lentine $\mathrm{KL}$, Costa SP, Weir MR, et al. Cardiac disease evaluation and management among kidney and liver transplantation candidates: a scientific statement from the American Heart Association and the American College of Cardiology Foundation: endorsed by the American Society of Transplant Surgeons, American Society of Transplantation, and National Kidney Foundation. Circulation 2012;126:617-63.

27. Ting SM, Hamborg T, McGregor G, et al. Reduced cardiovascular reserve in chronic kidney failure: a matched cohort study. Am J Kidney Dis 2015;66:274-84.

28. De Lima JJ, Sabbaga E, Vieira ML, et al. Coronary angiography is the best predictor of events in renal transplant candidates compared with noninvasive testing. Hypertension 2003;42:263-8.

29. Rabbat CG, Treleaven DJ, Russell JD, Ludwin D, Cook DJ. Prognostic value of myocardial perfusion studies in patients with end-stage renal disease assessed for kidney or kidneypancreas transplantation: a meta-analysis. J Am Soc Nephrol 2003;14:431-9.

30. Wang LW, Masson P, Turner RM, et al. Prognostic value of cardiac tests in potential kidney transplant recipients: a systematic review. Transplantation 2015;99:731-45.

31. Herzog CA, Ma JZ, Collins AJ. Poor long-term survival after acute myocardial infarction among patients on long-term dialysis. $\mathrm{N}$ Engl J Med 1998;339:799-805.

32. Beattie JN, Soman SS, Sandberg KR, et al. Determinants of mortality after myocardial infarction in patients with advanced renal dysfunction. Am J Kidney Dis 2001;37:1191-200 [Erratum in: Am J Kidney Dis 2001;38:701].

33. Fox CS, Muntner P, Chen AY, et al. Use of evidence-based therapies in short-term outcomes of ST-segment elevation myocardial infarction and non-ST-segment elevation myocardial infarction in patients with chronic kidney disease: a report from the National Cardiovascular Data Acute Coronary Treatment and Intervention Outcomes Network registry. Circulation 2010;121:357-65.

34. Marenzi G, Cabiati A, Assanelli E. Chronic kidney disease in acute coronary syndromes. World J Nephrol 2012;1:134-45.
35. Santopinto JJ, Fox KA, Goldberg RJ, et al. Creatinine clearance and adverse hospital outcomes in patients with acute coronary syndromes: findings from the global registry of acute coronary events (GRACE). Heart 2003;89:1003-8.

36. Granger CB, Goldberg RJ, Dabbous O, et al. Predictors of hospital mortality in the global registry of acute coronary events. Arch Intern Med 2003;163:2345-53.

37. Marenzi G, Cosentino N, Guastoni C. How to balance risks and benefits in the management of CKD patients with coronary artery disease. J Nephrol 2015;28:403-13.

38. Sedlis SP, Jurkovitz CT, Hartigan PM, et al. Optimal medical therapy with or without percutaneous coronary intervention for patients with stable coronary artery disease and chronic kidney disease. Am J Cardiol 2009;104:1647-53.

39. Mohandas R, Segal MS, Huo T, et al. Renal function and coronary microvascular dysfunction in women with symptoms/ signs of ischemia. PLoS One 2015;10:e0125374.

40. Montalescot G, Sechtem U, Achenbach S, et al. 2013 ESC guidelines on the management of stable coronary artery disease: the Task Force on the management of stable coronary artery disease of the European Society of Cardiology. Eur Heart J 2013;34:2949-3003.

41. Ng TM, Ackerbauer KA, Hyderi AF, Hshieh S, Elkayam U. Comparative effects of nesiritide and nitroglycerin on renal function, and incidence of renal injury by traditional and RIFLE criteria in acute heart failure. J Cardiovasc Pharmacol Ther 2012;17(1):79-85.

42. Di Lullo L, Bellasi A, Russo D, et al. Ivabradine, heart failure and chronic kidney disease. Nephrology@Point of Care 2015;1(1):e39-43.

43. Agrawal $\mathrm{H}$, Aggarwal $\mathrm{K}$, Littrell $\mathrm{R}$, et al. Pharmacological and non pharmacological strategies in the management of coronary artery disease and chronic kidney disease. Curr Cardiol Rev 2015;11(3):261-9.

44. Ishii $\mathrm{H}$, Toriyama $\mathrm{T}$, Aoyama $\mathrm{T}$, et al. Efficacy of oral nicorandil in patients with end-stage renal disease: a retrospective chart review after coronary angioplasty in Japanese patients receiving hemodialysis. Clin Ther 2007;29(1):110-22.

45. Liu W, Ming Q, Shen J, et al. Trimetazidine Prevention of Contrast-Induced Nephropathy in Coronary Angiography. Am J Med Sci 2015;350(5):398-402.

46. Basra SS, Tsai P, Lakkis NM. Safety and efficacy of antiplatelet and antithrombotic therapy in acute coronary syndrome patients with chronic kidney disease. J Am Coll Cardiol 2011;58:2263-9.

47. Palmer SC, Di Micco L, Razavian M, et al. Effects of antiplatelet therapy on mortality and cardiovascular and bleeding outcomes in persons with chronic kidney disease: a systematic review and meta-analysis. Ann Intern Med 2012;156:445-59.

48. Subherwal S, Bach RG, Chen AY, et al. Baseline risk of major bleeding in non-ST-segment-elevation myocardial infarction: the CRUSADE (Can Rapid risk stratification of Unstable angina patients Suppress ADverse outcomes with Early implementation of the ACC/AHA Guidelines) Bleeding Score. Circulation 2009;119:1873-82.

49. Berger AK, Duval S, Krumholz HM. Aspirin, beta-blocker, and angiotensin-converting enzyme inhibitor therapy in patients with end-stage renal disease and an acute myocardial infarction. J Am Coll Cardiol 2003;42:201-8.

50. Baigent $C$, Landray $M$, Leaper $C$, et al. First United Kingdom Heart and Renal Protection (UK-HARP-I) study: biochemical efficacy and safety of simvastatin and safety of low-dose aspirin in chronic kidney disease. Am J Kidney Dis 2005;45:473-84.

51. Keltai M, Tonelli M, Mann JF, et al. Renal function and outcomes in acute coronary syndrome: impact of clopidogrel. Eur J Cardiovasc Prev Rehabil 2007;14:312-8. 
52. Wiviott SD, Braunwald E, McCabe et al. Prasugrel versus clopidogrel in patients with acute coronary syndromes. N Engl J Med 2007;357:2001-15.

53. Melloni C, Cornel JH, Hafley G, et al. Impact of chronic kidney disease on long-term ischemic and bleeding outcomes in medically managed patients with acute coronary syndromes: Insights from the TRILOGY ACS Trial. Eur Heart J Acute Cardiovasc Care 2015.

54. Wallentin L, Becker RC, Budaj A, et al. Ticagrelor versus clopidogrel in patients with acute coronary syndromes. N Engl J Med 2009;361:1045-57.

55. James S, Budaj A, Aylward P, et al. Ticagrelor versus clopidogrel in acute coronary syndromes in relation to renal function: results from the Platelet Inhibition and Patient Outcomes (PLATO) trial. Circulation 2010;122:1056-67.

56. Spinler SA, Mahaffey KW, Gallup D, et al. Relationship between renal function and outcomes in high-risk patients with nonST-segment elevation acute coronary syndromes: results from SYNERGY. Int J Cardiol 2010;144(1):36-41.

57. Fox KA, Antman EM, Montalescot G, et al. The impact of renal dysfunction on outcomes in the ExTRACT-TIMI 25 trial. J Am Coll Cardiol 2007;49:2249-55.

58. Fox KA, Bassand JP, Mehta SR, et al. Influence of renal function on the efficacy and safety of fondaparinux relative to enoxaparin in non ST-segment elevation acute coronary syndromes. Ann Intern Med 2007;147:304-10.

59. Mehran R, Nikolsky E, Lansky AJ, et al. Impact of chronic kidney disease on early (30-day) and late (1-year) outcomes of patients with acute coronary síndromes treated with alternative antithrombotic treatment strategies: an ACUITY (Acute Catheterization and Urgent Intervention Triage strategY) substudy. JACC Cardiovasc Interv 2009;2:748-57.

60. Steg PG, James SK, Atar D, et al. ESC Guidelines for the management of acute myocardial infarction in patients presenting with ST-segment elevation. Eur Heart J 2012;33:2569-619.

61. Shlipak MG, Heidenreich PA, Noguchi H, Chertow GM, Browner WS, McClellan MB. Association of renal insufficiency with treatment and outcomes after myocardial infarction in elderly patients. Ann Intern Med 2002;137:555-62.

62. Afsar B, Turkmen K, Covic A, Kanbay M. An update on coronary artery disease and chronic kidney disease. Int J Nephrol 2014; 2014:767424.

63. Wali RK, Iyengar M, Beck GJ, et al. Efficacy and safety of carvedilol in treatment of heart failure with chronic kidney disease: a meta-analysis of randomized trials. Circ Heart Fail 2011; 4:18-26.

64. Cice G, Ferrara L, Di Benedetto A, et al. Dilated cardiomyopathy in dialysis patients-beneficial effects of carvedilol: a doubleblind, placebo-controlled trial. J Am Coll Cardiol 2001;2:407-11.

65. Wanner C, Krane V, März W, et al. Atorvastatin in patients with type 2 diabetes mellitus undergoing hemodialysis. N Engl J Med 2005;3:238-48.

66. Fellström BC, Jardine AG, Schmieder RE, et al. Rosuvastatin and cardiovascular events in patients undergoing hemodialysis. $\mathrm{N}$ Engl J Med 2009;14:1395-407.

67. Tonelli M, Isles C, Curhan GC, et al. Effect of pravastatin on cardiovascular events in people with chronic kidney disease. Circulation 2004;110:1557-63.

68. Dasari TW, Cohen DJ, Kleiman NS, et al. Statin therapy in patients with chronic kidney disease undergoing percutaneous coronary intervention (from the Evaluation of Drug Eluting Stents and
Ischemic Events Registry. Am J Cardiol 2014;113:621-5.

69. Baigent C, Landray MJ, Reith C, et al. The effects of lowering LDL cholesterol with simvastatin plus ezetimibe in patients with chronic kidney disease (Study of Heart and Renal Protection): a randomised placebo-controlled trial. Lancet 2011;377: 2181-92.

70. Ashrith G, Lee VV, Elayda MA, Reul RM, Wilson JM. Short- and long-term outcomes of coronary artery bypass grafting or drug-eluting stent implantation for multivessel coronary artery disease in patients with chronic kidney disease. Am J Cardiol 2010;106:348-53.

71. Gupta T, Paul N, Kolte D, et al. Associtation of chronic renal insufficiency with in-hospital outcomes after percutaneous coronary intervention. J Am Heart Assoc 2015;4:e002069.

72. Hemmelgarn BR, Southern D, Culleton BF, Mitchell LB, Knudtson $\mathrm{ML}$, Ghali WA. Survival after coronary revascularization among patients with kidney disease. Circulation 2004;110:1890-5.

73. Huang HD, Alam M, Hamzeh I, et al. Patients with severe chronic kidney disease benefit from early revascularization after acute coronary syndrome. Int J Cardiol 2013;168:3741-6.

74. Charytan D, Mauri L, Agarwal A, Servoss S, Scirica B, Kuntz RE. The use of invasive cardiac procedures after acute myocardial infarction in long-term dialysis patients. Am Heart J. 2006; 152:558-64.

75. Szummer $\mathrm{K}$, Lundman $\mathrm{P}$, Jacobson $\mathrm{SH}$, et al. Influence of renal function on the effects of early revascularization in non-STelevation myocardial infarction: data from the Swedish WebSystem for Enhancement and Development of Evidence-Based Care in Heart Disease Evaluated According to Recommended Therapies (SWEDEHEART). Circulation 2009;120:851-8.

76. Windecker S, Kolh P, Alfonso F, et al. 2014 ESC/EACTS Guidelines on myocardial revascularization: The Task Force on Myocardial Revascularization of the European Society of Cardiology and the European Association for Cardio-Thoracic Surgery, developed with the special contribution of the European Association of Percutaneous Cardiovascular Interventions. Eur Heart J 2014;35: 2541-619.

77. Best PJ, Berger PB, Davis BR, et al. Impact of mild or moderate chronic kidney disease on the frequency of restenosis: results from the PRESTO trial. J Am Coll Cardiol 2004;44:1786-91.

78. Sedlis SP, Jurkovitz CT, Hartigan PM, et al. Optimal medical therapy with or without percutaneous coronary intervention for patients with stable coronary artery disease and chronic kidney disease. Am J Cardiol 2009;104:1647-53.

79. Medi C, Montalescot G, Budaj A, et al. Reperfusion in patients with renal dysfunction after presentation with ST-segment elevation or left bundle branch block: GRACE (Global Registry of Acute Coronary Events). JACC Cardiovasc Interv 2009;2:26-33.

80. Ix JH, Mercado N, Shlipak MG, et al. Association of chronic kidney disease with clinical outcomes after coronary revascularization: the Arterial Revascularization Therapies Study (ARTS). Am Heart J 2005;149:512-9.

81. Marui A, Kimura T, Nishiwaki N, et al. Percutaneous coronary intervention versus coronary artery bypass grafting in patients with end-stage renal disease requiring dialysis (5-year outcomes of the CREDO-Kyoto PCI/CABG Registry Cohort-2). Am J Cardiol 2014;114:555-61.

82. Zheng $H$, Xue S, Lian F, Huang RT, Hu ZL, Wang YY. Meta-analysis of clinical studies comparing coronary artery bypass grafting with percutaneous coronary intervention in patients with endstage renal disease. Eur J Cardiothorac Surg 2013;43:459-67. 